\title{
Isolation of Escherichia coli Strains with AcrAB-TolC Efflux Pump-Associated Intermediate Interpretation or Resistance to Fluoroquinolone, Chloramphenicol and Aminopenicillin from Dogs Admitted to a University Veterinary Hospital
}

\author{
Toyotaka SATO ${ }^{1)}$, Shin-ichi YOKOTA ${ }^{2)}$, Risa ICHIHASHI ${ }^{1)}$, Tomoka MIYAUCHI ${ }^{1)}$, Torahiko OKUBO ${ }^{1)}$, Masaru USUI ${ }^{1)}$, \\ Nobuhiro FUJII' ${ }^{2)}$ and Yutaka TAMURA ${ }^{1) *}$ \\ ${ }^{1)}$ Laboratory of Food Microbiology and Food Safety, Department of Health and Environmental Sciences, School of Veterinary Medicine, \\ Rakuno Gakuen University, 582 Midorimachi, Bunkyodai, Ebetsu 069-8501, Hokkaido, Japan \\ ${ }^{2)}$ Department of Microbiology, Sapporo Medical University School of Medicine, South-1, West-17, Chuo-ku, Sapporo, Hokkaido \\ 060-8556, Japan
}

(Received 22 March 2013/Accepted 3 March 2014/Published online in J-STAGE 18 March 2014)

ABSTRACT. Understanding the prevalence of antimicrobial-resistance and the relationship between emergence of resistant bacteria and clinical treatment can facilitate design of effective treatment strategies. We here examined antimicrobial susceptibilities of Escherichia coli isolated from dogs admitted to a university hospital (University hospital) and companion animal clinics (Community clinics) in the same city and investigated underlying multidrug-resistance mechanisms. The prevalence of $E$. coli with intermediate and resistant interpretations to ampicillin (AMP), enrofloxacin (ENR) and chloramphenicol (CHL) was higher in the University hospital than in the Community clinics cases. Use of antimicrobials, including fluoroquinolone, was also significantly higher in the University hospital than in the Community clinics cases. Upon isolation using ENR-supplemented agar plates, all ENR-resistant isolates had 3-4 nucleotide mutations that accompanied by amino acid substitutions in the quinolone-resistance-determining regions of $\operatorname{gyr}$, parC and parE, and $94.7 \%$ of all isolates derived from the University hospital showed AMP and/or CHL resistance and possessed bla $a_{\mathrm{TEM}}$ and/or catAl. The average mRNA expression levels of $a \mathrm{cr} A, \operatorname{acr} B$ and tolC and the prevalence of organic solvent tolerance, in isolates derived from ENR-supplemented agar plates were significantly higher in the University hospital than in the Community clinics isolates. Thus, E. coli derived from the University hospital cases more often showed concomitant decreased susceptibilities to aminopenicillins, fluoroquinolones and CHL than did those derived from the Community clinics; this was related to an active AcrAB-TolC efflux pump, in addition to acquisition of specific resistance genes and genetic mutations.

KEY WORDS: AcrAB, antimicrobial resistance, canine, efflux pump, Escherichia coli.

doi: 10.1292/jvms.13-0144; J. Vet. Med. Sci. 76(7): 937-945, 2014

Excessive and inappropriate use of antimicrobial agents leads to the generation and spread of antimicrobial-resistant bacteria $[1,26]$. Resistant bacteria encountered in companion animal medicine also represent a potential hazard to human health $[2,23,25]$, because companion animals live in close proximity with humans and receive medical treatment, including antimicrobials used for humans [22].

Understanding the prevalence of antimicrobial resistance and the mechanisms involved allows estimation of the association between the emergence of resistant bacteria and clinical treatments. This is important for devising effective treatment strategies against bacterial infections in companion animals and for reducing the risk of transmission of antimicrobial-resistant bacteria from companion animals to humans.

\footnotetext{
*Correspondence to: Tamura, Y., Laboratory of Food Microbiology and Food Safety, Department of Health and Environmental Sciences, School of Veterinary Medicine, Rakuno Gakuen University,582 Midorimachi Bunkyodai Ebetsu, Hokkaido 069-8501, Japan. e-mail: tamuray@rakuno.ac.jp

(C)2014 The Japanese Society of Veterinary Science

This is an open-access article distributed under the terms of the Creative Commons Attribution Non-Commercial No Derivatives (by-nc-nd) License $<$ http://creativecommons.org/licenses/by-nc-nd/3.0/>.
}

Medical treatment of companion animals consists of primary medical care in companion animal clinics in the community and secondary medical care in university-related veterinary hospitals. Generally, university-related veterinary hospitals favor heavier and/or more frequent exposure to antimicrobial agents in seriously ill animals. Most previous surveillance studies of antimicrobial resistance in companion animals have taken place either in the community or in university hospitals; previous studies typically did not distinguish between or compare these settings [7, 22, 25] and mostly did not clarify the actual antimicrobials used. Therefore, comprehensive surveillance, including obtaining information on actual antimicrobial use, should be carried out in both primary and secondary medical care settings within the same region to investigate the extent of antimicrobial resistance in companion animals.

Fluoroquinolones are broad-spectrum antimicrobials and are amongst the most important antimicrobial agents used to treat a variety of bacterial infections, not only in humans, but also in companion animals. Thus, emergence of fluoroquinolone-resistant bacteria due to antimicrobial treatment may present a serious challenge in clinical treatment of bacterial infections $[2,8]$. Therefore, surveillance of fluoroquinoloneresistant bacteria could offer important information for the control of infectious diseases. 
Fluoroquinolone resistance is mainly caused by chromosomal mutations in the quinolone-resistance-determining region (QRDR) of topoisomerase IV and DNA gyrase [3, 6, 10, 29]. Moreover, plasmid-mediated quinolone-resistance genes (PMQRs), such as qnr, aac (6')-Ib-cr and qepA, have been reported in Gram-negative bacteria, including $E$. coli [12]. Furthermore, the overexpression of efflux pumps, mainly AcrAB-TolC, in E. coli concomitantly decreases susceptibility to fluoroquinolones [19]. Detailed investigations of these fluoroquinolone resistance mechanisms are important for elucidating the differences in mechanisms underlying emergence of fluoroquinolone-resistant $E$. coli isolates between primary and secondary medical care and could provide beneficial information for controlling $E$. coli infection in each type of facility.

In this study, we examined the antimicrobial-susceptibility of $E$. coli isolates derived from dogs sampled in a university hospital and in community companion animal clinics located in the same city. We also investigated the multidrugresistance mechanisms involved, including AcrAB-TolC function.

\section{MATERIALS AND METHODS}

Clinical histories and condition of host dogs: In total, 173 cotton rectal swabs were collected from 93 dogs treated at Rakuno Gakuen University (RGU) Veterinary Teaching Hospital (Ebetsu, Japan; University hospital) and from 80 dogs treated at 8 companion animal clinics (10 samples per clinic, from different dogs) in the community of Ebetsu (Community clinics) from June to December 2005 (regardless of the clinical condition seen for the animal). All dogs admitted to the University hospital had also visited the Community clinics previously.

University hospital cases (15 male and 20 female dogs) included those with tumor, cataract, glaucoma, keratitis, hip dysplasia, Cushing syndrome and herniated intervertebral discs. Community clinic cases (27 male and 24 female dogs) included those undergoing castration, panovario-hysterectomy or treatment for urinary tract infections, cystitis, chronic diarrhea, dermatitis, otitis externa, gingivitis, pharyngitis and keratitis. Dogs were aged 0-16 years (University dogs: $8.2 \pm 3.7$ y [mean \pm SD]; Community dogs: $5.5 \pm 4.2$ y). The 6-month history of antimicrobial use prior to sampling was also compared for the 54 dogs admitted to the University hospital and the 56 dogs admitted to the Community clinics.

Bacterial isolates: Canine rectal samples were collected before commencing clinical treatment. Samples were streaked on deoxycholate-hydrogen sulfide-lactose (DHL) agar (Nissui, Tokyo, Japan) and incubated for $24 \mathrm{hr}$ at $37^{\circ} \mathrm{C}$. Colonies of suspected E. coli growing on these DHL agar plates were picked and subcultured on nutrient agar (Nissui). After incubation, the biochemical properties of these colonies were assessed using triple sugar iron agar (Nissui), lysine indole motility medium (Nissui) and cytochrome oxidase tests. Final identification of $E$. coli was performed using API20E codes (bioMérieux, Tokyo, Japan). The 173 canine samples were also assessed on DHL agar supplemented with
$4 \mu \mathrm{g} / \mathrm{m} l$ of enrofloxacin (ENR; Bayer, Osaka, Japan).

Susceptibility testing: Susceptibilities to a panel of antimicrobials were examined using the agar dilution method, according to the guidelines of the Clinical and Laboratory Standards Institute (CLSI) [5]. Mueller-Hinton (MH) agar was obtained from Oxoid (Basingstoke, U.K.). Ampicillin (AMP), amoxicillin (AMX), cefazolin (CFZ), cephalexin (LEX), gentamicin (GEN), kanamycin (KAN), dihydrostreptomycin (DSM), oxytetracycline (OTC) and chloramphenicol (CHL) were obtained from Sigma-Aldrich (St. Louis, MO, U.S.A.), and cefpodoxime (CPD) was purchased from Daiichi Sankyo Co., Ltd. (Tokyo, Japan). Staphylococcus aureus ATCC29213, Enterococcus faecalis ATCC29212, E. coli ATCC25922 and Pseudomonas aeruginosa ATCC27853 were used as controls. Resistant, intermediate and susceptible interpretations were as defined by CLSI guidelines [5]. Resistance to DSM $(\geq 32 \mu \mathrm{g} / \mathrm{m} l)$ and OTC $(\geq 16 \mu \mathrm{g} / \mathrm{m} l)$ was microbiologically defined as described in the Japanese Veterinary Antimicrobial-resistance Monitoring System [18]. Intermediate interpretations for DSM and OTC were defined as having two-fold lower minimum inhibitory concentration (MIC) than those of the resistance category. Phe-Arg- $\beta$ naphthylamide $(\mathrm{PA} \beta \mathrm{N}$; Sigma-Aldrich; final concentration: $20 \mu \mathrm{g} / \mathrm{m} l$ ) was used as an efflux-pump inhibitor [24].

Organic solvent tolerance: Organic solvent tolerance (OST) was investigated as previously described [28] with slight modifications. An overnight culture of E. coli was diluted with $0.9 \% \mathrm{NaCl}$ (approximately $1 \times 10^{7}$ cells $/ \mathrm{m} l$ ). A drop of cell suspension $(5 \mu l)$ was spotted onto $\mathrm{MH}$ agar medium to form a circle with a diameter of $8 \mathrm{~mm}$. The surface of the agar was overlaid with a mixture $(3: 1,1: 1$, or $1: 3$ [vol/ vol]) of $n$-hexane ( $96.0 \%$ pure; Kishida Chemical Co., Ltd., Osaka, Japan) and cyclohexane (>99\% pure; Merck KGaA, Darmstadt, Germany) to a depth of $3 \mathrm{~mm}$. Cyclohexane is an organic solvent known to be a more effective agent against $E$. coli than $n$-hexane [28]. Bacterial growth was assessed after the plates were incubated at $37^{\circ} \mathrm{C}$ for $16-18 \mathrm{hr}$ in a sealed vessel. Confluent growth of the cells (confluent) was considered to be indicative of tolerance to the solvent tested. When only a few colonies $(<10)$ grew on the plate or when no growth was observed, the cells were considered to be sensitive to the solvent tested (non-confluent). Each experiment was performed 3 times, and averages were calculated.

Determination of QRDR mutations, PMQRs, $\beta$-lactamases and CHL-resistance genes: Mutations in QRDRs of gyrA, parC, parE and $g y r B$ were examined by direct DNA sequencing of PCR products, as described by Everett et al. [9]. PMQR genes (qnrA, qnrB, qnrS, aac (6') Ib-cr and qepA) were detected by PCR using specific primers (Table 1 ) and direct DNA sequencing $[4,15,21]$. To identify the AMPresistance mechanism, $\beta$-lactamase genes, viz., $b l a_{\mathrm{TEM}}$ and $b l a_{\mathrm{SHV}}$, were detected by PCR and direct DNA sequencing [16]. CHL-resistance genes, viz., catA1, catA2, catA3, floR and $c m l A$, were detected by PCR as described in previous studies $[17,27]$. Nucleotide sequences were determined using a BigDye Terminator v3.1 Cycle Sequencing Kit with a 3130 Genetic Analyzer (Applied Biosystems, Foster City, CA, U.S.A.). 


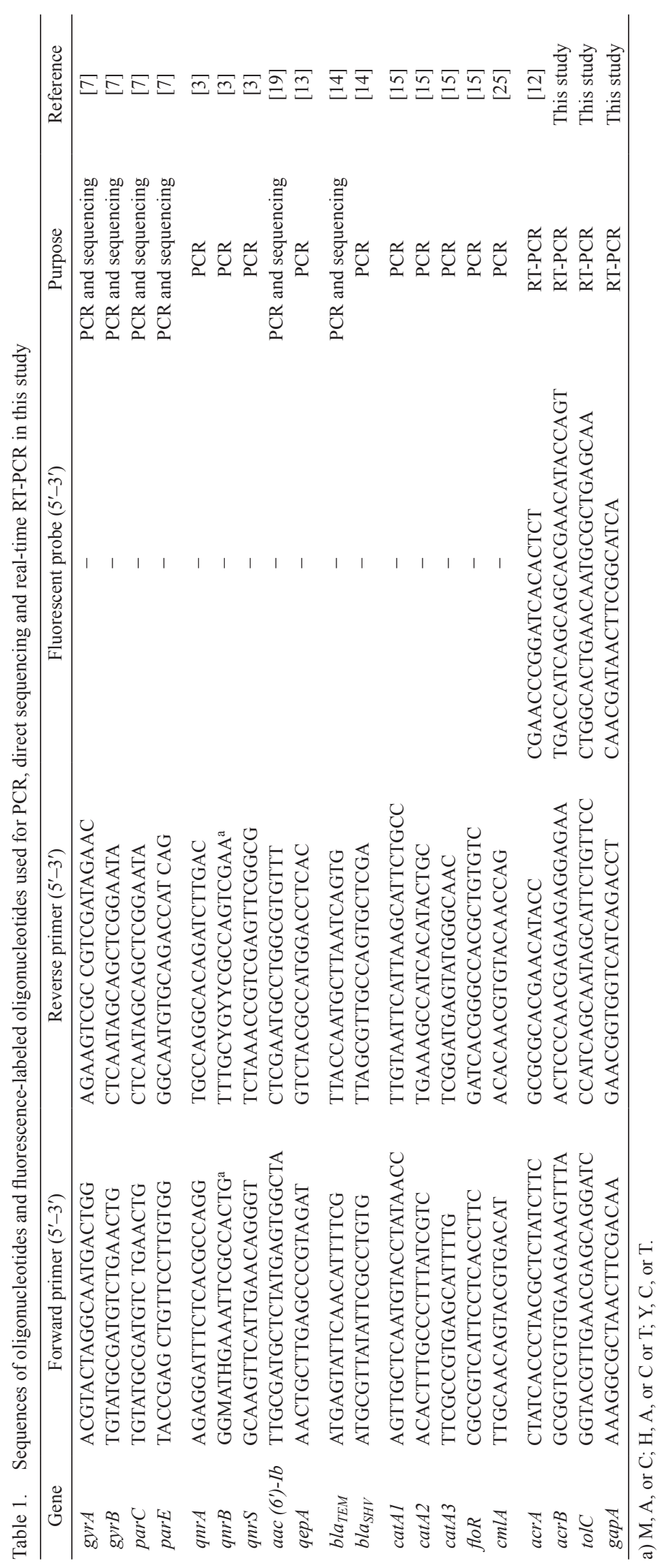


Table 2. Antimicrobial susceptibility of E. coli strains derived from dogs attending Rakuno Gakuen University Veterinary Teaching Hospital (RGU; University) and animal clinics in the community (Community)

\begin{tabular}{|c|c|c|c|c|c|c|c|c|}
\hline \multirow{2}{*}{$\begin{array}{c}\text { Antimicrobial } \\
\text { (break point, } \mu \mathrm{g} / \mathrm{m} l \text { ) }\end{array}$} & \multirow{2}{*}{ Groups } & \multirow{2}{*}{$\begin{array}{l}\text { Range } \\
(\mu \mathrm{g} / \mathrm{m} l)\end{array}$} & \multirow{2}{*}{$\begin{array}{l}\mathrm{MIC}_{50} \\
(\mu \mathrm{g} / \mathrm{m} l)\end{array}$} & \multirow{2}{*}{$\begin{array}{l}\mathrm{MIC}_{90} \\
(\mu \mathrm{g} / \mathrm{m} l)\end{array}$} & \multicolumn{4}{|c|}{ Number of strains (\%) } \\
\hline & & & & & $\mathrm{S}$ & I & $\mathrm{R}$ & $\mathrm{I}+\mathrm{R}$ \\
\hline \multirow{2}{*}{$\begin{array}{l}\text { AMP } \\
(\geq 32)\end{array}$} & University & $2->128$ & 4 & $>128$ & $44(59.5)^{*}$ & $4(5.4)$ & $26(35.1)$ & $30(40.5)^{*}$ \\
\hline & Community & $0.5->128$ & 4 & $>128$ & $50(75.8)$ & 0 & $16(24.2)$ & $16(24.2)$ \\
\hline \multirow{2}{*}{$\begin{array}{l}\text { AMX } \\
(\geq 32)\end{array}$} & University & $1->128$ & 16 & $>128$ & $32(43.2)^{* *}$ & $15(20.3)^{* *}$ & $27(36.5)$ & $42(56.8)^{* *}$ \\
\hline & Community & $4->128$ & 4 & $>128$ & $50(75.8)$ & 0 & $16(24.2)$ & $16(24.2)$ \\
\hline \multirow{2}{*}{$\begin{array}{c}\text { CFZ } \\
(\geq 32)\end{array}$} & University & $1->128$ & 2 & $>128$ & $61(82.4)$ & $1(1.4)$ & $12(16.2)$ & $13(17.6)$ \\
\hline & Community & $1->128$ & 2 & $>128$ & $57(86.3)$ & 0 & $9(13.6)$ & $9(13.6)$ \\
\hline \multirow{2}{*}{$\begin{array}{l}\text { LEX } \\
(\geq 32)\end{array}$} & University & $8->128$ & 8 & $>128$ & $54(73.0)$ & $5(6.8)$ & $15(20.3)$ & $20(27.0)$ \\
\hline & Community & $4->128$ & 8 & $>128$ & 47 (71.2) & $9(13.6)$ & $10(15.1)$ & $19(28.8)$ \\
\hline \multirow{2}{*}{$\begin{array}{l}\text { CPD } \\
(\geq 8)\end{array}$} & University & $<0.125->128$ & 0.5 & 128 & $62(83.8)$ & 0 & $12(16.2)$ & $12(16.2)$ \\
\hline & Community & $0.25->128$ & 0.5 & 128 & $57(86.4)$ & 0 & $9(13.6)$ & $9(13.6)$ \\
\hline \multirow{2}{*}{$\begin{array}{l}\text { KAN } \\
(\geq 64)\end{array}$} & University & $1->128$ & 2 & 32 & $66(89.2)$ & $1(1.4)$ & $7(9.5)$ & $8(10.8)$ \\
\hline & Community & $2->128$ & 2 & $>128$ & $59(89.4)$ & 0 & 7 (10.6) & 7 (10.6) \\
\hline \multirow{2}{*}{$\begin{array}{l}\text { GEN } \\
(\geq 16)\end{array}$} & University & $0.5->128$ & 1 & 8 & $67(90.5)$ & 0 & $7(9.5)$ & $7(9.5)$ \\
\hline & Community & $0.5-64$ & 1 & 2 & $62(93.9)$ & 0 & $4(6.1)$ & $4(6.1)$ \\
\hline \multirow{2}{*}{$\begin{array}{l}\text { DSM } \\
(\geq 32)\end{array}$} & University & $2->128$ & 4 & $>128$ & $55(74.3)$ & $1(1.4)$ & $18(24.3)$ & $19(25.7)$ \\
\hline & Community & $2->128$ & 4 & $>128$ & $48(72.7)$ & $1(1.5)$ & $17(25.8)$ & $18(27.3)$ \\
\hline \multirow{2}{*}{$\begin{array}{l}\text { OTC } \\
(\geq 16)\end{array}$} & University & $2->128$ & 2 & $>128$ & $56(75.7)$ & $3(4.1)$ & $15(20.3)$ & $18(24.3)$ \\
\hline & Community & $1->128$ & 2 & $>128$ & $50(75.8)$ & 0 & $16(24.2)$ & $16(24.2)$ \\
\hline \multirow{2}{*}{$\begin{array}{l}\text { CHL } \\
(\geq 32)\end{array}$} & University & $4->128$ & 8 & 64 & $54(73.0)^{*}$ & $9(12.2)$ & $11(14.9)^{*}$ & $20(27.0)^{*}$ \\
\hline & Community & $4->128$ & 8 & 8 & $61(92.4)$ & $3(4.5)$ & $2(3.0)$ & $5(7.6)$ \\
\hline \multirow{2}{*}{$\begin{array}{l}\text { ENR } \\
(\geq 4)\end{array}$} & University & $0.01-128$ & 0.03 & 64 & $59(79.7)^{*}$ & 0 & $15(20.3)^{*}$ & $15(20.3)^{*}$ \\
\hline & Community & $0.01-64$ & 0.03 & 16 & $61(92.4)$ & 0 & $5(7.5)$ & $5(7.6)$ \\
\hline
\end{tabular}

AMP: Ampicillin, AMX: Amoxicillin, CFZ: Cefazolin, CHL: Chloramphenicol, CPD: Cefpodoxime, DSM: Dihydrostreptomycin, ENR: Enrofloxacin, GEN: Gentamicin, KAN: Kanamycin, LEX: Cephalexin, OTC: Oxytetracycline. S: Susceptible, I: Intermediate, R: Resistant. ${ }^{*} P<0.05$, ${ }^{* *} P<0.01$; difference versus Community.

Table 3. Prevalence of concomitant antimicrobial resistance on ENR-resistant E. coli isolates derived from non-supplemented agar

\begin{tabular}{lllllllllll}
\hline \multirow{2}{*}{ Isolates } & \multicolumn{10}{c}{ Prevalence of concomitant resistance (\%) } \\
\cline { 2 - 11 } & AMP & AMX & CEZ & LEX & CPD & KAN & GEN & DSM & OTC & CHL \\
\hline ENR-resistant (20) & $90.0^{* * *}$ & $90.0^{* *}$ & $75.0^{* *}$ & $80.0^{* *}$ & $70.0^{* *}$ & 15.0 & $30.0^{* *}$ & $65.0^{* *}$ & 35.0 & $40.0^{* *}$ \\
ENR-susceptible (120) & 20.0 & 20.8 & 5.0 & 7.5 & 5.8 & 9.2 & 4.2 & 18.3 & 20.0 & 2.5 \\
\hline
\end{tabular}

AMP: Ampicillin, AMX: Amoxicillin, CFZ: Cefazolin, CHL: Chloramphenicol, CPD: Cefpodoxime, DSM: Dihydrostreptomycin, ENR: Enrofloxacin, GEN: Gentamicin, KAN,:Kanamycin, LEX: Cephalexin, OTC: Oxytetracycline. ${ }^{* * P<0.01 .}$

Real-time reverse transcription- $P C R$ : Overnight cultures of $E$. coli isolates were diluted 1:100 in LB broth and grown to the mid-logarithmic phase. RNA was isolated using an RNeasy Mini kit (Qiagen, Hilden, Germany) according to the manufacturer's instructions and stored at $-80^{\circ} \mathrm{C}$ until used. The concentration of RNA was determined spectrophotometrically (BioSpectrometer, Eppendorf, Hamburg, Germany). Gene expression ( $a c r A, a c r B$ and tolC) was estimated by quantitative reverse transcription (RT) TaqManPCR. The respective primer pairs and probes (Table 1) used for $a \operatorname{cr} B$, tolC and gap $A$ in this study were designed according to the sequence of $E$. coli strain K12 substrain MG1655, which is deposited in GenBank (accession number U00096). The probes were labeled by the manufacturer (Sigma-Aldrich) with the reporter dye 6-carboxyfluorescein (6'-FAM) at the $5^{\prime}$-end and with the quencher dye 6-carboxytetramethylrhodamine (TAMRA) at the 3 '-end. Purified RNA (2.5 ng) was used in one-step RT and real-time PCR amplification. RT-PCR amplification mixtures $(20 \mu l)$ contained purified RNA, $2 \times$ QuantiTect Probe RT-PCR Master Mix, $0.2 \mu l$ of QuantiTect RT Mix (QuantiTect Probe RT-PCR kit, Qiagen), $0.2 \mu \mathrm{M}$ of probe and $0.5 \mu \mathrm{M}$ forward and reverse primers. The cycle conditions comprised 20-min reverse transcription at $50^{\circ} \mathrm{C}$; a 15 -min initial activation step at $95^{\circ} \mathrm{C}$; and 45 cycles each of $55^{\circ} \mathrm{C}$ for $1 \mathrm{~min}$ and at $60^{\circ} \mathrm{C}$ for $30 \mathrm{sec}$ in a LightCycler 480 (Roche, Mannheim, Germany). Expression of gapA was used to normalize expression ratios. E. coli strain AG100 (K-12 argE3 thi-1 rpsL xyl mtl D (gal-uvrB) supE44) was kindly donated by Dr Helen I. Zgurskaya (University of Oklahoma, Norman, OK, U.S.A.) and used as a reference strain. All experiments were performed 3 times, and averages were calculated.

Statistical analysis: Statistical significance of differences between the isolates obtained from dogs admitted to the 2 
Table 4. Organic solvent tolerance (OST) of E. coli strains derived from dogs in attending Rakuno Gakuen University Veterinary Teaching Hospital (RGU; University) and animal clinics in the community (Community)

\begin{tabular}{cccl}
\hline $\begin{array}{c}\text { OST } \\
(n \text {-hexane: } \\
\text { cyclohexane })\end{array}$ & Groups & Non-confluent & Confluent \\
\hline $3: 1$ & University & $32(43.2)^{* *}$ & $42(56.8)^{* *}$ \\
& Community & $55(83.3)$ & $11(16.7)$ \\
$1: 1$ & University & $60(81.1)^{* *}$ & $14(18.9)^{* *}$ \\
& Community & $63(95.5)$ & $3(4.5)$ \\
$1: 3$ & University & $67(90.5)$ & $7(9.5)^{*}$ \\
& Community & $65(98.5)$ & $1(1.5)$ \\
\hline
\end{tabular}

Values indicate the number of $E$. coli isolates and (percentage of the total). ${ }^{*} P<0.05, * * P<0.01$; statistical difference versus Community.

types of treatment facilities was determined by Student's $t$-test and Fisher's exact test. $P$-value $<0.05$ was considered statistically significant.

\section{RESULTS}

Antimicrobial-resistance profile of canine E. coli isolates: There was a significant difference in the ages $(P<0.05)$, but not in the gender distribution of the dogs admitted to the University hospital or the Community clinics.

Seventy-four E. coli isolates were obtained from 93 rectal samples from dogs admitted to the University hospital $(79.6 \%)$ and 66 isolates from 80 rectal samples obtained from dogs admitted to the Community clinics (82.5\%), after culture on DHL agar plates that had not been supplemented with ENR. There was no significant difference in the frequency of $E$. coli isolation between dogs admitted to the 2 types of treatment facilities $(P>0.05)$.

Of all the canine $E$. coli isolates, $44.3 \%$ (62 of 140 isolates) were resistant to at least 1 antimicrobial agent tested with aminopenicillin resistance being the most frequent (approximately $30 \%$ ); approximately $50 \%$ of aminopenicillinresistant isolates were also resistant to cephalosporins (CFZ and CPD). Although there was no significant difference in the rate of resistance to AMP or AMX between isolates derived from University hospital cases and isolates derived from Community clinics cases, when considering isolates with resistance as well as those with an intermediate interpretation to AMP and AMX, this rate was significantly more prevalent in the University hospital than in the Community clinics samples $(P<0.05$, Table 2$)$. The prevalence of CHLresistant and ENR-resistant isolates was also significantly higher in the University hospital than in the Community clinics samples (Table 2).

In terms of susceptibilities to aminopenicillin and CHL among the 15 ENR-resistant isolates derived from the University hospital samples, 7 isolates showed resistance and/ or intermediate interpretation to aminopenicillin, as well as resistance to CHL. Six of these isolates showed resistance and/or intermediate interpretation to aminopenicillin, but susceptibility to $\mathrm{CHL}$, while the remaining 2 isolates showed
Table 5. Status of antimicrobial use in dogs attending Rakuno Gakuen University Veterinary Teaching Hospital (RGU; University) and animal clinics in the community (Community)

\begin{tabular}{lcc}
\hline \multicolumn{1}{c}{ Antimicrobial use } & University & Community \\
\hline $\begin{array}{l}\text { Average number of antimicrobials } \\
\text { used for each dog }\end{array}$ & $1.4^{* *}$ & 0.8 \\
$\begin{array}{l}\text { Frequency of dogs treated } \\
\text { by fluoroquinolone }\end{array}$ & $24.1 \% * *$ & $14.3 \%$ \\
$\begin{array}{l}\text { Frequency of dogs treated } \\
\text { by all antimicrobials }\end{array}$ & $74.1 \% * *$ & $50.0 \%$ \\
\hline
\end{tabular}

We could obtain antimicrobial use history for 6 months prior to sampling from 54 dogs in the University and 56 dogs in the Community. **P<0.01; statistical difference versus Community.

susceptibility to both aminopenicillin and CHL (data not shown). Among the 5 ENR-resistant isolates derived from the Community clinics samples, 1 isolate showed both resistance to aminopenicillin and CHL, and 4 isolates showed resistance and/or an intermediate interpretation to aminopenicillin, but susceptibility to CHL (data not shown). The prevalence of resistance to aminoglycosides (KAN, GEN and DSM) and OTC was not significantly different between isolates derived from the University hospital cases and from the Community clinics cases. ENR-resistant isolates frequently demonstrated concomitant resistance to aminopenicillins, cephalosporins, GEN, DSM and CHL (Table 3). Prevalence of OST was significantly higher in isolates from the University hospital cases than from the Community clinics cases (Table 4).

The average number of antimicrobials used for each dog was significantly higher in the University hospital than in the Community clinics cases (Table 5). The frequencies of dogs treated with any antimicrobials and with fluoroquinolones were also significantly higher in the University hospital than in the Community clinics cases (Table 5). In addition, prevalence of fluoroquinolone-resistant isolates was significantly higher in dogs that had been treated with fluoroquinolones compared with that in dogs that had not been treated with this agent $(P<0.05$; data not shown).

Isolation of fluoroquinolone-resistant E. coli using ENRsupplemented DHL agar plates: To investigate fluoroquinolone-resistance mechanisms and the occurrence of multidrug resistance involving fluoroquinolone, we selected ENRresistant E. coli on ENR-supplemented DHL agar plates (Fig. 1). Rate of resistance to aminopenicillins, cephalosporins, GEN, DSM, OTC and CHL was significantly higher in isolates obtained from ENR-supplemented DHL agar plates than those obtained from DHL agar plates that had not been supplemented with ENR. Isolates obtained from ENR-supplemented DHL agar plates were most frequently AMP resistant.

We further characterized the $31 \mathrm{E}$. coli isolates derived from ENR-supplemented DHL agar plates (Table 6). All ENR-resistant isolates had nucleotide substitutions in QRDRs accompanied by changes in 3 or 4 amino acids in QRDRs. The $a a c$ (6') $\mathrm{Ib}-\mathrm{cr}$, one of the genes encoding PMQRs, was detected in only 1 strain. In total, more than $70 \%$ of 


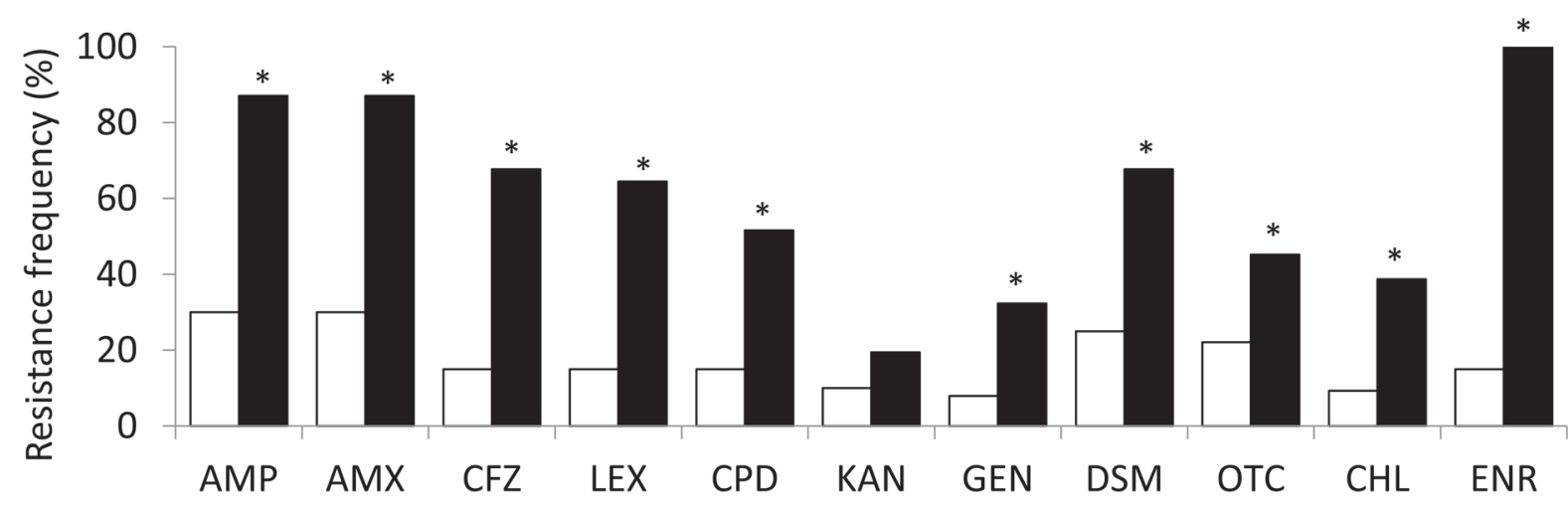

Fig. 1. Influence of enrofloxacin selection on isolation frequencies of $E$. coli isolated from canine rectal samples. AMP, ampicillin; AMX, amoxicillin; CFZ, cefazolin; CHL, chloramphenicol; CPD, cefpodoxime; DSM, dihydrostreptomycin; ENR, enrofloxacin; GEN, gentamicin; KAN, kanamycin; LEX, cephalexin; OTC, oxytetracycline. *Statistical difference for isolation with deoxycholate hydrogen sulfide lactose (DHL) medium without antimicrobials; $P<0.05$.

the ENR-resistant isolates had resistance or intermediate interpretation to AMP and/or CHL, and $74 \%$ of isolates with an AMP MIC of $>128 \mu \mathrm{g} / \mathrm{m} l$ possessed $b l a_{\mathrm{TEM}-1 \text {, and }}$ $100 \%$ of isolates with a CHL MIC of $>128 \mu \mathrm{g} / \mathrm{m} l$ possessed catA1(Table 6). Expression levels of $a c r A$, acrB and tolC and the effect of PA $\beta \mathrm{N}$ were higher in CHL-resistance and CHL-intermediate interpretable isolates than in CHLsusceptible isolates (Table 6). Isolates exhibiting OST had high $\operatorname{acr} B$ expression, while isolates with an intermediate interpretation to AMP also exhibited OST and had higher $a c r B$ expression than did isolates that were AMP-susceptible (data not shown).

Among dogs from which we isolated E. coli on ENR-supplemented DHL agar plates, the frequency of dogs treated with any antimicrobials was significantly higher in the University hospital $(89.5 \%)$ than in the Community clinics $(58.3 \%)$ cases (Table 6). In contrast, the frequency of dogs treated with fluoroquinolones was not significantly different between the University hospital $(31.6 \%)$ and Community clinics $(25.0 \%)$ cases. Twenty-seven of $31 \mathrm{E}$. coli isolates obtained on ENR-supplemented DHL agar plates showed resistance or an intermediate interpretation to AMP and/or CHL. Among the 27 dogs from which we isolated E. coli with resistant or an intermediate interpretation to AMP and/ or CHL on ENR-supplemented DHL agar plates, 18 dogs had been treated with fluoroquinolone and/or $\beta$-lactam antimicrobials (Table 6).

\section{DISCUSSION}

In this study, E. coli isolates with resistant or an intermediate interpretation to aminopenicillins, CHL or fluoroquinolone were more frequently obtained from dogs admitted to the Universal hospital than from those admitted to the Community clinics. Remarkably, isolates with resistance to fluoroquinolones more frequently showed resistance to aminopenicillins, cephalosporins, GEN, DSM and CHL, as compared with fluoroquinolone-susceptible isolates. This result suggested that the difficulty of providing effective antimicrobial treatment increases in secondary medical care. It indicated a need to investigate the mechanism underlying the emergence of this multidrug-resistance phenotype.

To characterize in detail the fluoroquinolone-resistant isolates obtained from the University hospital and Community clinics studied here, we investigated antimicrobial-resistance mechanisms of $E$. coli isolates derived from dogs using ENR-supplemented DHL agar plates. All ENR-resistant isolates obtained from ENR-supplemented DHL agar plates possessed 3 or 4 mutations in QRDRs. A previous study showed that in vitro exposure to fluoroquinolone caused mutations in QRDRs and AcrAB-TolC overexpression [13]. This may indicate that in vivo fluoroquinolone exposure can also cause an increase in fluoroquinolone-resistant E. coli possessing multiple mutations in QRDRs and AcrAB-TolC overexpression. Indeed, prevalence of fluoroquinolone-resistant isolates was significantly higher in dogs that had been treated with fluoroquinolones compared with that in dogs that had not been treated with this agent, as determined using on DHL agar plates that had not been supplemented with ENR. Moreover, fluoroquinolone-resistant isolates derived from the University hospital had higher levels of $a c r A, a c r B$ and tolC expression than did such isolates obtained from the Community clinics, as determined using ENR-supplemented DHL agar plates. These findings suggested that the high prevalence of fluoroquinolone-resistant $E$. coli isolates derived from the University hospital may have been caused by frequent fluoroquinolone use in the University hospital and/ or continuous fluoroquinolone use in the Community clinics and the University hospital. This may have resulted in development of a mechanism that decreased fluoroquinolone susceptibility, viz., overexpression of AcrAB-TolC.

In this study, CHL, in addition to ENR was another agent to which isolates derived from the University hospital showed a significantly higher prevalence of resistance than 


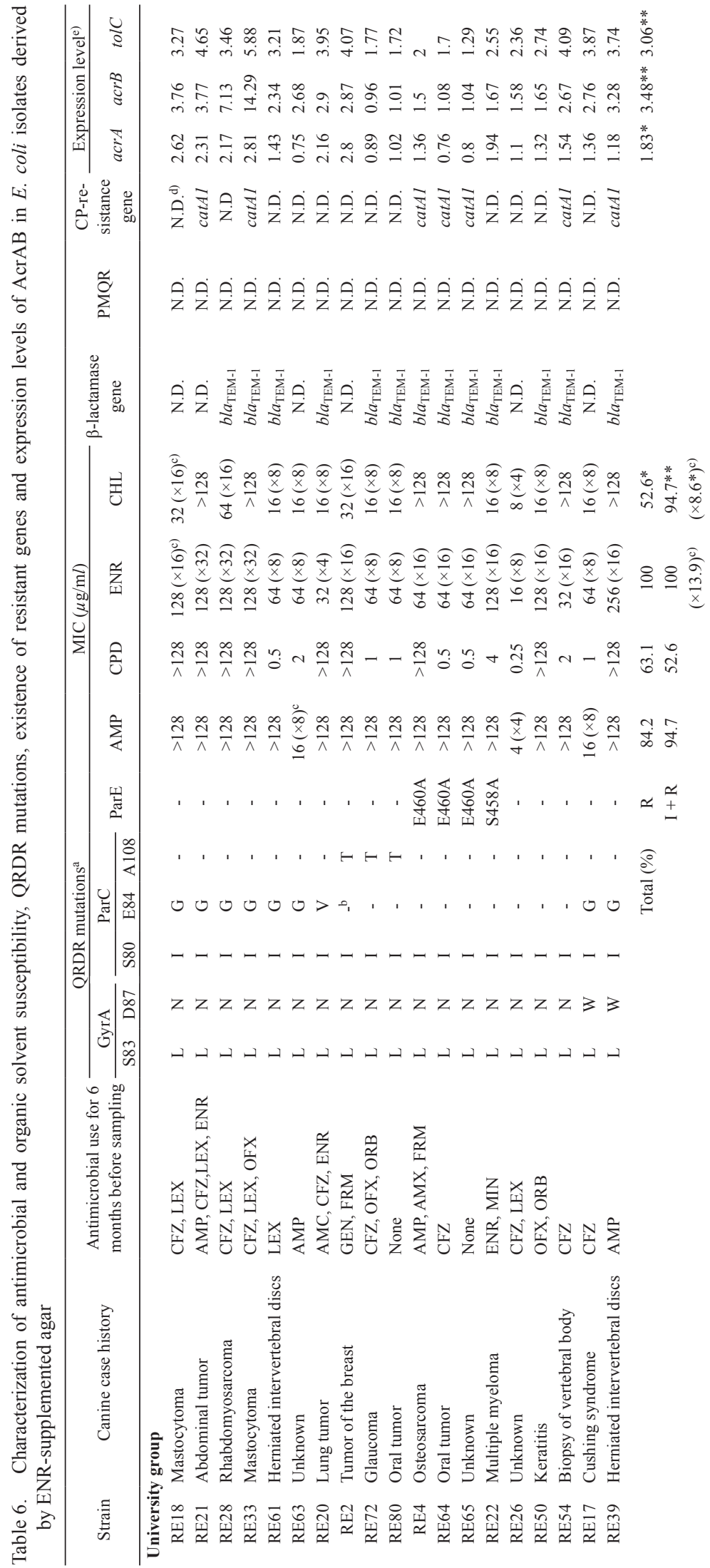

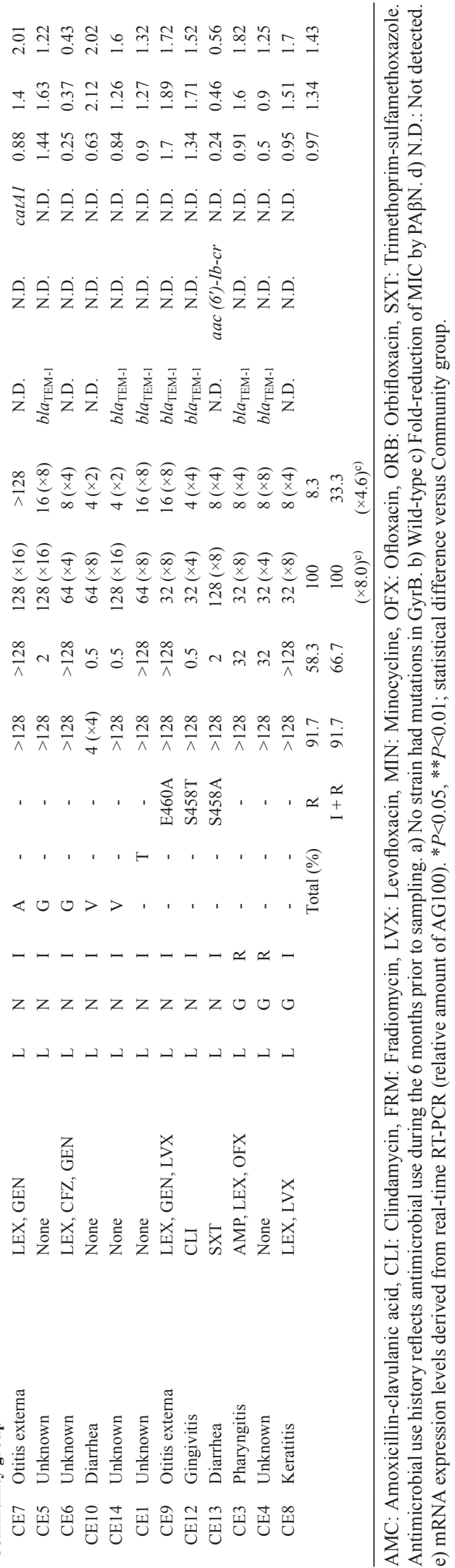


did those derived from the Community clinics. All ENR-resistant isolates with a CHL MIC of $>128 \mu \mathrm{g} / \mathrm{m} l$ that had been derived from ENR-supplemented DHL agar plates possessed catA1. However, other resistant isolates with a CHL MIC of 32 and $64 \mu \mathrm{g} / \mathrm{ml}$ and an intermediate interpretation isolates with a CHL MIC of $16 \mu \mathrm{g} / \mathrm{m} l$ did not possess any specific CHL-resistance gene. Among all antimicrobial agents that we tested, isolates with ENR resistance were most frequently co-resistant to aminopenicillins, and all the isolates showing resistance to AMP, but not to cepharosporins, possessed blaTEM-1. However, isolates with intermediate interpretation to AMP did not possess any of the $\beta$-lactamase genes for which we tested. These results indicated that the main resistance mechanisms for fluoroquinolones, AMP and CHL involved by acquisition of mutations in QRDRs and a resistance-associated gene, e.g., bla $a_{\mathrm{TEM}-1}$ or catA1, although there may also be other mechanisms that decreased their susceptibilities and conferred co-resistance to these agents.

To evaluate the mechanism underlying decreased susceptibilities and co-resistance to fluoroquinolone, aminopenicillins and $\mathrm{CHL}$, we investigated AcrAB-TolC function, because AcrAB-TolC is a major resistance-nodulation-division (RND) family-type efflux pump that excretes several antimicrobials $[14,19,20]$. AcrAB overexpression increases the MICs of aminopenicillins and CHL to an intermediate interpretation $(16 \mu \mathrm{g} / \mathrm{m} l)$ or resistance $(32$ or $64 \mu \mathrm{g} / \mathrm{m} l)$ level, and its effect is not limited to fluoroquinolone resistance $[13,21]$. AcrAB-TolC is also known to cause the efflux of several organic solvents, which cause cell death by breaking down microbial membranes [11]; therefore, investigation of OST is useful for identifying $E$. coli isolates that have active AcrAB-TolC [28]. We observed that OST isolates with higher $a c r B$ expression and isolates with an intermediate interpretation to aminopenicillins and CHL, as well as isolates resistant to aminopenicillins and/or with CHL MICs of 32 and $64 \mu \mathrm{g} / \mathrm{ml}$, also exhibited OST and higher $a c r B$ expression than did susceptible isolates, as seen by analysis using ENR-supplemented DHL agar plates. A higher prevalence of isolates with OST, decreased aminopenicillin susceptibility and decreased CHL susceptibility, was observed in isolates obtained from University hospital compared to those from Community clinics cases, as seen on agar plates without ENR supplementation. These results supported the notion that active AcrAB-TolC function contributes to a decrease in susceptibility to aminopenicillins and CHL MICs in some E. coli isolates obtained from dogs.

Our study revealed that the frequency of total antimicrobial treatment as well as fluoroquinolone use was significantly higher in the University hospital than in the Community clinics. This evidence suggested that the frequent use of antimicrobials in dogs admitted to the University hospital and/ or their continuous use in dogs moving from the Community clinics to the University hospital facilitate selection of antimicrobial resistant E. coli strains with QRDR mutations, beta-lactamase gene and catAl. In addition, our study also revealed that dogs admitted to the University hospital tend to be treated with multiple antimicrobials. This approach may facilitate development of multidrug-resistant $E$. coli isolates.
Indeed, our results showed that ENR-resistant E. coli isolates had higher rates of resistance to several antimicrobials compared with ENR-susceptible E. coli isolates, and ENR-resistant isolates derived from the University hospital cases on ENR-supplemented DHL agar showed a stronger development of the AcrAB-TolC than did ENR-resistant $E$. coli isolates derived from the Community clinics cases. We considered that these findings substantially reflect the situation in Japanese companion animal medicine, because the samples in this study were successively. In addition, a previous study also showed that AMP or ENR treatment led to the emergence of aminopenicillin-ENR-CHL-resistant E. coli isolates in dogs in the United States [2, 8]. Moreover, fluoroquinolone-aminopenicillin-CHL-resistant $E$. coli isolates with overexpression of AcrAB-TolC were frequently isolated from humans in university hospitals [28]. These findings indicate that the emergence of this multidrug-resistant phenotype may mirror the same phenomenon in human and companion animal clinical fields in several countries in some cases. In these cases, a clearer strategy for choice and use of antimicrobials suitable to treatments is required in order to prevent the emergence and spread of these fluoroquinoloneresistant $E$. coli with decreased susceptibilities to several other antimicrobials. In particular, we suggest that it may be important to share the history of antimicrobials usage across the first and secondary medical care settings of companion animals to avoid treatment with several antimicrobials in the same period and to avoid extensive, continuous treatment with the same class antimicrobial.

In conclusion, this study revealed that the higher prevalence of concomitant resistant and intermediate interpretations to fluoroquinolones, aminopenicillins and CHL in isolates from the University hospital than in isolates from the Community clinics was due not only to the acquisition of specific resistance mechanisms, such as $\beta$-lactamases, catA1 and QRDR mutations, but also to overexpression of the AcrAB-TolC efflux pump in canine E. coli.

ACKNOWLEDGMENTS. We thank Dr Helen I. Zgurskaya (University of Oklahoma, Norman, OK, U.S.A.) for providing E. coli strain AG100. This study was supported in part by a grant for developing the supporting system for upgrading education and research from the Japan Ministry of Education, Culture, Sports, Science and Technology, and the Japanese Ministry of Health, Labor and Welfare (H21Shokuhin-Japan-013).

\section{REFERENCES}

1. Aarestrup, F. M. 1999. Association between the consumption of antimicrobial agents in animal husbandry and the occurrence of resistant bacteria among food animals. Int. J. Antimicrob. Agents 12: 279-285. [Medline] [CrossRef]

2. Aly, S. A., Debavalya, N., Suh, S. J., Oryazabal, O. A. and Boothe, D. M. 2012. Molecular mechanisms of antimicrobialresistance in fecal Escherichia coli of healthy dogs after enrofloxacin or amoxicillin administration. Can. J. Microbiol. 58: 1288-1294. [Medline] [CrossRef]

3. Breines, D. M., Ouabdesselam, S., Ng, E. Y., Tankovic, J., Shah, 
S., Soussy, C. J. and Hooper, D. C. 1997. Quinolone resistance locus $n f x D$ of Escherichia coli is a mutant allele of the parE gene encoding a subunit of topoisomerase IV. Antimicrob. Agents Chemother. 41: 175-179. [Medline]

4. Cattoir, V., Poirel, L., Rotimi, V., Soussy, C. J. and Nordmann, P. 2007. Multiplex PCR for detection of plasmid-mediated quinolone resistance $q n r$ genes in ESBL-producing enterobacterial isolates. J. Antimicrob. Chemother. 60: 394-397. [Medline] [CrossRef]

5. Clinical and Laboratory Standards Institute. 2011. Methods for Dilution Antimicrobial Susceptibility Tests for Bacteria that Grow Aerobically; Approved Standard. 8th ed., CLSI document M100-S21. Wayne, PA.

6. Conrad, S., Oethinger, M., Kaifel, D., Klots, G., Marre, R. and Kern, W. V. 1996. gyrA mutations in high-level fluoroquinoloneresistant clinical isolates of Escherichia coli. J. Antimicrob. Chemother. 38: 443-455. [Medline] [CrossRef]

7. Costa, D., Poeta, P., Sáenz, Y., Coelho, A. C., Matos, M., Vinué, L., Rodrigues, J. and Torres, C. 2008. Prevalence of antimicrobial-resistance and resistance genes in faecal Escherichia coli isolates recovered from healthy pets. Vet. Microbiol. 127: 97-105. [Medline] [CrossRef]

8. Dawn, M. B. and Nipattra, D. 2011. Impact of routine antimicrobial therapy on canine fecal Escherichia coli antimicrobial-resistance: A pilot study. Intern. J. Appl. Res. Vet. Med. 9: 396-406.

9. Everett, M. J., Jin, Y. F., Ricci, V. and Piddock, L. J. 1996. Contributions of individual mechanisms to fluoroquinolone-resistance in 36 Escherichia coli isolated from humans and animals. Antimicrob. Agents Chemother. 40: 2380-2386. [Medline]

10. Heisig, P. 1996. Genetic evidence for a role of $\operatorname{parC}$ mutations in development of high-level fluoroquinolone-resistance in Escherichia coli. Antimicrob. Agents Chemother. 40: 879-885. [Medline]

11. Isken, S. and de Bont, J. A. 1998. Bacteria tolerant to organic solvents. Extremophiles 2: 229-238. [Medline] [CrossRef]

12. Jacoby, G. A. 2005. Mechanisms of resistance to quinolones. Clin. Infect. Dis. 41 Suppl. 2: S120-S126. [Medline] [CrossRef]

13. Jellen-Ritter, A. S. and Kern, W. V. 2001. Enhanced expression of the multidrug efflux pumps AcrAB and AcrEF associated with insertion element transposition in Escherichia coli mutants Selected with a fluoroquinolone. Antimicrob. Agents Chemother. 45: 1467-1472. [Medline] [CrossRef]

14. Keeney, D., Ruzin, A., McAleese, F., Murphy, E. and Bradford, P. A. 2008. MarA-mediated overexpression of the AcrAB efflux pump results in decreased susceptibility to tigecycline in Escherichia coli. J. Antimicrob. Chemother. 61: 46-53. [Medline] [CrossRef]

15. Kim, H. B., Park, C. H., Kim, C. J., Kim, E. C., Jacoby, G. A. and Hooper, D. C. 2009. Prevalence of plasmid-mediated quinolone resistance determinants over a 9-year period. Antimicrob. Agents Chemother. 53: 639-645. [Medline] [CrossRef]

16. Kojima, A., Ishii, Y., Ishihara, K., Esaki, H., Asai, T., Oda, C., Tamura, Y., Takahashi, T. and Yamaguchi, K. 2005. Extendedspectrum-beta-lactamase-producing Escherichia coli strains isolated from farm animals from 1999 to 2002: Report from the Japanese Veterinary Antimicrobial-resistance Monitoring program. Antimicrob. Agents Chemother. 49: 3533-3537. [Medline] [CrossRef]

17. Maynard, C., Fairbrother, J. M., Bekal, S., Sanschagrin, F.,
Levesque, R. C., Brousseau, R., Masson, L., Larivière, S. and Harel, J. 2003. Antimicrobial-resistance genes in enterotoxigenic Escherichia coli O149:K91 isolates obtained over a 23-year period from pigs. Antimicrob. Agents Chemother. 47: 3214-3221. [Medline] [CrossRef]

18. National Veterinary Assay Laboratory, Ministry of Agriculture, Forestry and Fisheries. 2009. A report on the Japanese Veterinary Antimicrobial-resistance Monitoring System -2000 to 2007. [http://www.maff.go.jp/nval/tyosa_kenkyu/taiseiki/pdf/ jvarm2000_2007_final_201005.pdf].

19. Nikaido, H. 1998. Antibiotic resistance caused by gram-negative multidrug efflux pumps. Clin. Infect. Dis. 27 Suppl. 1: S32-S41. [Medline] [CrossRef]

20. Okusu, H., Ma, D. and Nikaido, H. 1996. AcrAB efflux pump plays a major role in the antibiotic resistant phenotype of Escherichia coli multiple-antibiotic-resistance (Mar) mutants. J. Bacteriol. 178: 306-308. [Medline]

21. Park, C. H., Robicsek, A., Jacoby, G. A., Sahm, D. and Hooper, D. C. 2006. Prevalence in the United States of aac (6')-Ib-cr encoding a ciprofloxacin-modifying enzyme. Antimicrob. Agents Chemother. 50: 3953-3955. [Medline] [CrossRef]

22. Pedersen, K., Pedersen, K., Jensen, H., Finster, K., Jensen, V. F. and Heuer, O. E. 2007. Occurrence of antimicrobial-resistance in bacteria from diagnostic samples from dogs. J. Antimicrob. Chemother. 60: 775-781. [Medline] [CrossRef]

23. Phillips, I., Casewell, M., Cox, T., De Groot, B., Friis, C., Jones, R., Nightingale, C., Preston, R. and Waddell, J. 2004. Dose the use of antibiotics in food animals pose a risk to human health? A critical review of published data. J. Antimicrob. Chemother. 53: 28-52. [Medline] [CrossRef]

24. Sáenz, Y., Ruiz, J., Zarazaga, M., Teixidó, M., Torres, C. and Vila, J. 2004. Effect of the efflux pump inhibitor Phe-Arg-betanaphthylamide on the MIC values of the quinolones, tetracycline and chloramphenicol, in Escherichia coli isolates of different origin. J. Antimicrob. Chemother. 53: 544-545. [Medline] [CrossRef]

25. Sanchez, S., McCrackin Stevenson, M. A., Hudson, C. R., Maier, M., Buffington, T., Dam, Q. and Maurer, J. J. 2002. Characterization of multidrug-resistant Escherichia coli isolates associated with nosocomial infections in dogs. J. Clin. Microbiol. 40: 3586-3595. [Medline] [CrossRef]

26. Tamura, Y. 2003. Trends in antimicrobial agents for veterinary use and control measures of antimicrobial-resistance. J. Jpn. Vet. Med. 56: 685-691.

27. Travis, R. M., Gyles, C. L., Reid-Smith, R., Poppe, C., McEwen, S. A., Friendship, R., Janecko, N. and Boerlin, P. 2006. Chloramphenicol and kanamycin resistance among porcine Escherichia coli in Ontario. J. Antimicrob. Chemother. 58: 173-177. [Medline] [CrossRef]

28. Wang, H., Dzink-Fox, J. L., Chen, M. and Levy, S. B. 2001. Genetic characterization of highly fluoroquinolone-resistant clinical Escherichia coli strains from China: role of acrR mutations. Antimicrob. Agents Chemother. 45: 1515-1521. [Medline] [CrossRef]

29. Yoshida, H., Bogaki, M., Nakamura, M., Yamanaka, L. Y. and Nakamura, S. 1991. Quinolone resistance-determining region in the DNA gyrase gyrB gene of Escherichia coli. Antimicrob. Agents Chemother. 35: 1647-1650. [Medline] [CrossRef] 